\title{
A Case of Primary Small Bowel Melanoma Diagnosed by Single-Balloon Enteroscopy
}

\author{
Jun Young Shin ${ }^{1}$, In Suh Park², Byoung Wook Bang ${ }^{1}$, Hyung Kil Kim¹, Yong Woon Shin ${ }^{1}$ and Kye Sook Kwon ${ }^{1}$ \\ ${ }^{1}$ Division of Gastroenterology, Department of Internal Medicine, ${ }^{2}$ Department of Pathology, Inha University School of Medicine, Incheon, \\ Korea
}

Although metastasis from cutaneous malignant melanoma to the small intestine is not uncommon, primary small bowel melanoma (SBM) is extremely rare. This case report describes a rare case of primary SBM, diagnosed by single-balloon enteroscopy. A 74-yearold man presented with recurrent melena. Upper endoscopy and colonoscopy were unremarkable. Abdominal computed tomography (CT) revealed an ileal mass with ileo-ileal intussusception. Subsequent single-balloon enteroscopy identified an ileal tumor, which was histologically diagnosed as melanoma. Extensive clinical examination did not reveal any primary cutaneous lesions. To the best of our knowledge, this is the first case of primary SBM in South Korea. Clin Endosc 2017;50:395-399

Key Words: Intestine, small; Melanoma; Single-balloon enteroscopy

\section{INTRODUCTION}

Malignant melanomas constitute $1 \%-3 \%$ of all malignant tumors of the gastrointestinal (GI) tract. ${ }^{1,2}$ However, the majority of these tumors are secondary lesions representing metastatic spread of a primary tumor. ${ }^{3}$ Primary small bowel melanoma (SBM) is very rare; approximately 30 cases of SBM have been reported worldwide, although there is some debate as to whether SBM arises as a true small bowel primary tumor or metastasizes from unidentified or regressed primary cutaneous melanomas. ${ }^{4}$ Clinically, both primary and metastatic SBMs are more aggressive than other melanoma subtypes and have worse prognoses. ${ }^{1}$

Like other small bowel tumors, SBM is typically difficult to diagnose, owing to its nonspecific symptoms and challenging

Received: November 11, 2016 Revised: March 7, 2017

Accepted: April 27, 2017

Correspondence: Byoung Wook Bang

Division of Gastroenterology, Department of Internal Medicine, Inha University Hospital, Inha University School of Medicine, 27 Inhang-ro, Jung-gu, Incheon 22332, Korea

Tel: +82-32-890-2548, Fax: +82-32-890-2549, E-mail: bangbu@inha.ac.kr

(cc) This is an Open Access article distributed under the terms of the Creative Commons Attribution Non-Commercial License (http://creativecommons.org/ licenses/by-nc/3.0) which permits unrestricted non-commercial use, distribution, and reproduction in any medium, provided the original work is properly cited. endoscopic approach. A final diagnosis is invariably achieved postoperatively. However, the introduction of single-balloon enteroscopy enables accurate diagnosis of small bowel tumors prior to surgery. We experienced a very unusual case of primary SBM, confirmed by single-balloon enteroscopy.

\section{CASE REPORT}

A 74-year-old man was admitted to our hospital with anemia and intermittent melena. He had multiple underlying medical conditions including hypertension, atrial fibrillation, coronary heart disease, and a history of cerebral infarction. At the time of his admission, he was taking multiple medications, including antihypertensives, an antihyperlipidemic agent, and an anticoagulant. He had been admitted two months prior to the current admission, also because of melena and anemia. A series of studies performed at that time, including abdominal computed tomography (CT), upper endoscopy, and colonoscopy, were unremarkable. The patient experienced no further bleeding during that hospital stay, and was discharged without further investigation. He had subsequently undergone regular checkups in the outpatient clinic, and was taking an iron supplement, but the anemia was not corrected. 


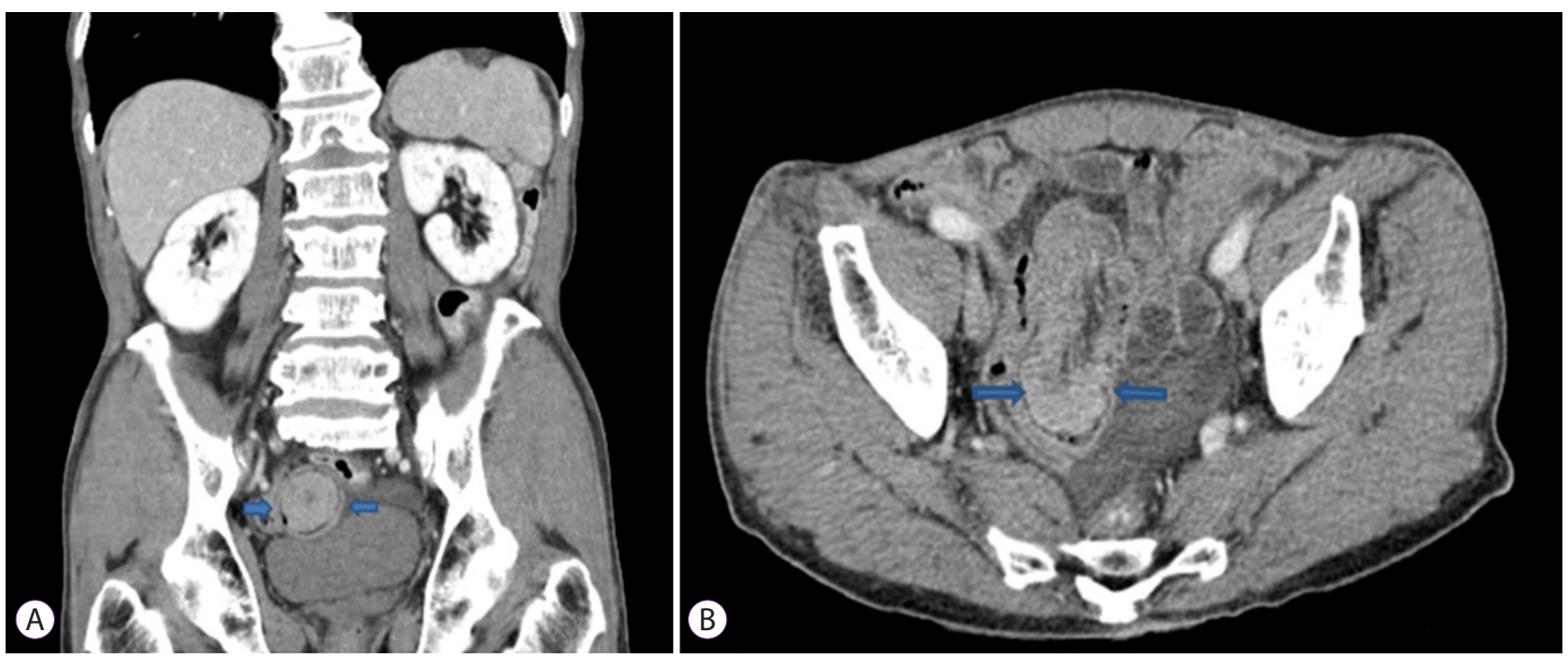

Fig. 1. Abdominal computed tomography (CT) showing $4 \mathrm{~cm}$ polypoid enhancing mass with ileo-ileal intussusception in the distal ileum (arrow). (A) Coronal image; (B) Axial image.

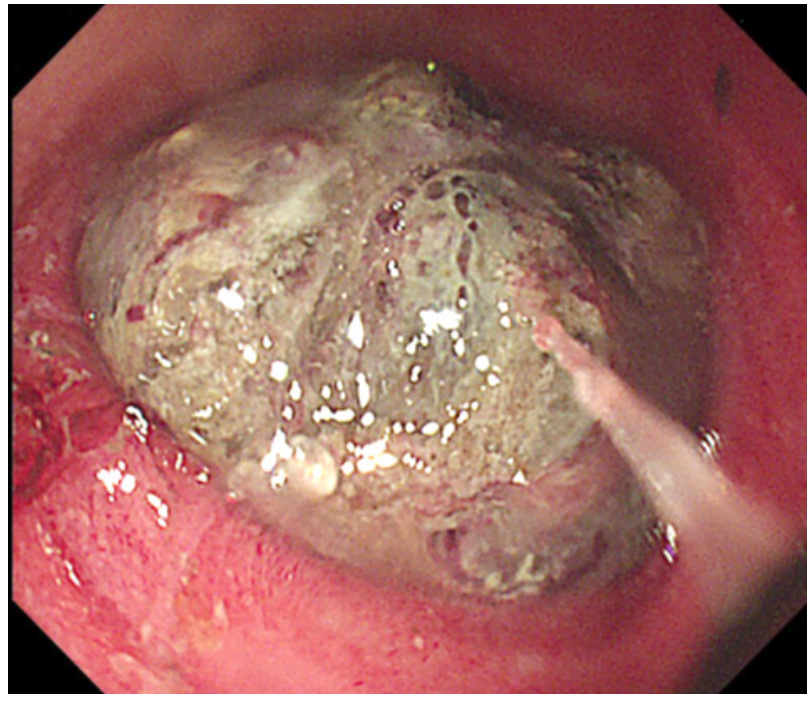

Fig. 2. Single-balloon enteroscopy showing $4 \mathrm{~cm}$ darkish polypoid mass obstructing the lumen approximately $60 \mathrm{~cm}$ proximal to the ileocecal valve.

Upon his current hospital admission, the patient's physical examination was unremarkable with the exception of general weakness. Laboratory investigations were within normal limits except for the anemia (hemoglobin $[\mathrm{Hb}] 5.9 \mathrm{~g} / \mathrm{dL}$ ). Upper endoscopy and colonoscopy were repeated, but failed to identify the bleeding focus. An abdominal CT scan demonstrated suspicious soft tissue with ileo-ileal intussusception in the distal ileum (Fig. 1). Single-balloon enteroscopy was performed and identified a $4 \mathrm{~cm}$ darkish polypoid ileal mass, approximately $60 \mathrm{~cm}$ proximal to the ileocecal valve (Fig. 2). Endoscopic biopsies were taken and India ink was injected around the tumor for preoperative localization. On histologic examination, the biopsy specimen revealed an infiltration of large atypical cells with prominent nucleoli and melanin pigment. Immunohistochemically, these cells were positive for HMB45 and S-100 protein. The biopsy diagnosis confirmed malignant melanoma (Fig. 3). Given the above findings, a thorough investigation of the skin, nose, oral mucosa, and anus was performed to detect unidentified cutaneous lesions, but failed to reveal any significant lesions. The patient underwent small bowel resection with end-to-end anastomosis and mesenteric lymph node dissection. Macroscopically, the tumor appeared as a darkish polypoid mass $4 \mathrm{~cm}$ in size originating in the distal ileum (Fig. 4A), and the tumor was the leading point of the ileo-ileal intussusception. Pathologic examination of resected specimens revealed that the tumor originated from the wall of the small intestine. The tumor cell findings were the same as those for the biopsy specimens, with lymphovascular invasion and tumor metastasis in five out of 27 mesenteric lymph nodes (Fig. 4). After surgery, a positron emission tomography (PET) scan was performed to reveal any hidden primary focus, but the scan was negative for hypermetabolic activity. A final diagnosis of primary ileal malignant melanoma with regional lymph node involvement was confirmed.

The patient was referred to the oncology department for adjuvant chemotherapy, but chemotherapy was not administered owing to his poor general condition. At this point, he has undergone regular follow-up visits at the outpatient clinic for one year, without experiencing recurrence.

\section{DISCUSSION}

Malignant melanoma is a rare tumor accounting for $1 \%-3 \%$ of all tumors. ${ }^{1,2}$ It is a malignant tumor derived from melano- 

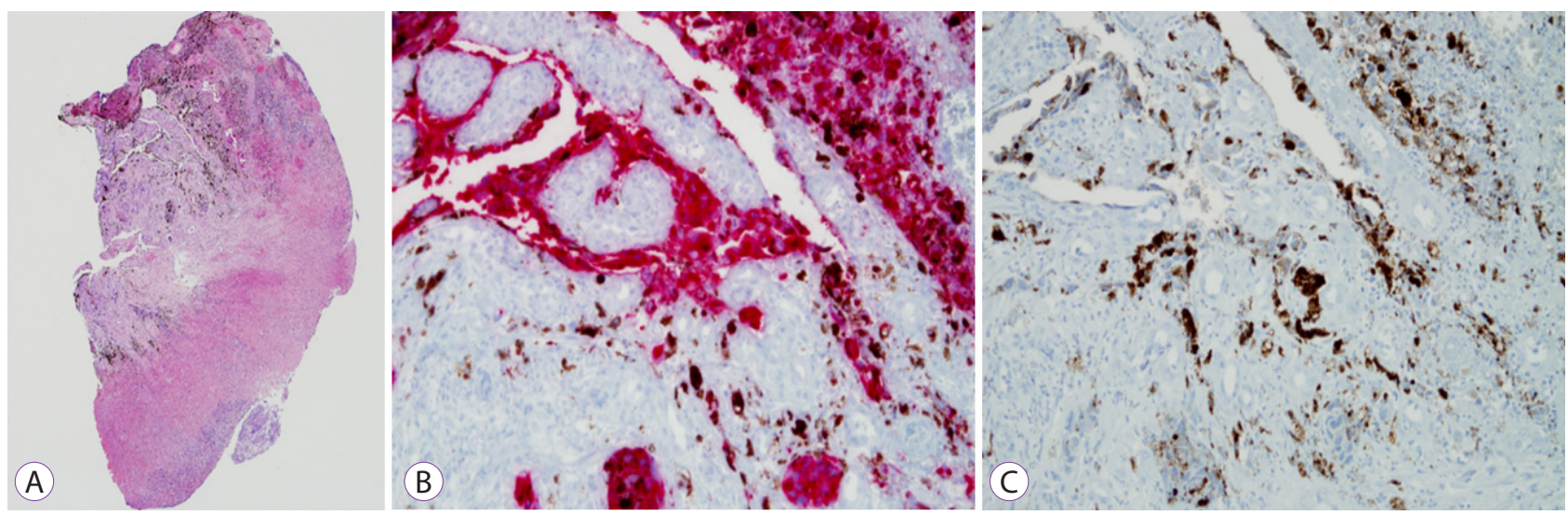

Fig. 3. Histologic examination of endoscopic biopsy specimen showing infiltration of large atypical cells with prominent nucleoli and melanin pigment (A) and positive immunohistochemical staining for HMB-45 and S-100 (B,C). (A) Hematoxylin and eosin staining (H\&E), $\times 40$. (B) Immunohistochemical staining for HMB-45 (red color) (×200). (C) Immunohistochemical staining for S-100 protein (brown color) $(\times 200)$.
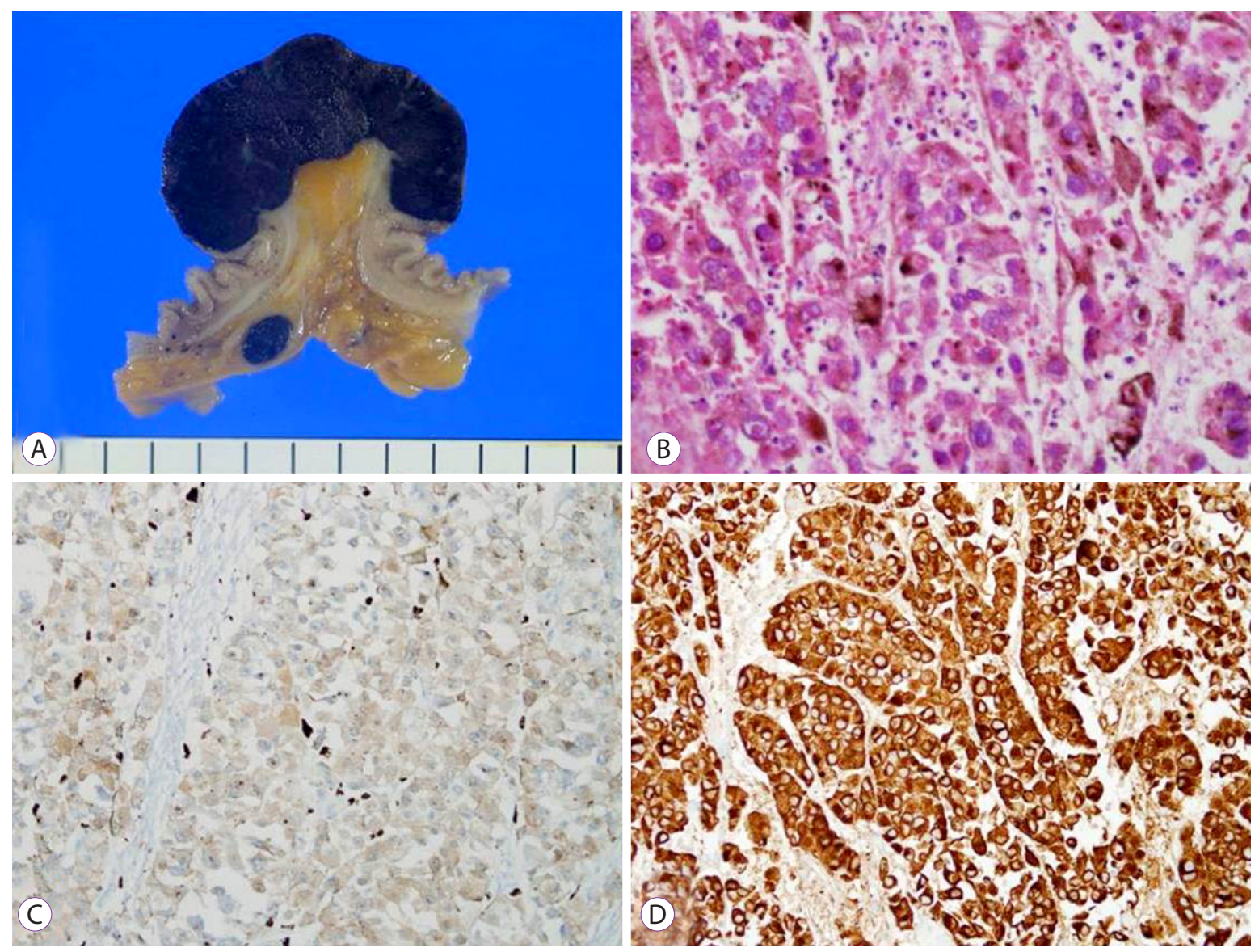

Fig. 4. Gross and histologic examination of surgical specimen. (A) Gross findings showed $4 \mathrm{~cm}$ darkish polypoid ileal mass with regional darkish lymph node. (B) Melanin pigment is observed in the tumor cells (Hematoxylin and eosin staining [H\&E], $\times 400)$. (C) Immunohistochemical staining for S-100 protein is positive ( $\times 200)$. (D) Immunohistochemical staining for HMB-45 is positive $(\times 200)$.

cytes. Melanocytes are dendritic-shaped melanin-producing cells crucial to skin pigmentation and ultraviolet protection in the skin and eye. ${ }^{5}$ They are present in anatomic sites including the skin, eye, and epithelia of the nasal cavity, oropharynx, bronchus, anus, vagina, and urinary tract. Although melano- ma is mostly of cutaneous origin, noncutaneous tumors can also occur, albeit very rarely. ${ }^{5}$

The small intestine is the most frequent site of metastatic melanoma in the digestive tract, because of its rich blood and lymphatic supply. ${ }^{2,6}$ Even though GI tract metastases are 
observed in $50 \%-60 \%$ of cases of malignant melanoma at autopsy, clinical evidence of GI involvement is found with ante-mortem diagnosis in only $1 \%-5 \%$ of cases. ${ }^{7}$ Melanoma can metastasize to any portion of the digestive tract, from mouth to anus, but the most common sites are the jejunum and ileum.

Primary SBM is extremely rare. To date, approximately 30 cases of primary SBM have been reported worldwide. ${ }^{4}$ SBM is rarely diagnosed at an early stage, tends to be more aggressive, and is associated with a poor prognosis. ${ }^{8}$ Men seem to be more frequently affected than women, with a male-to-female ratio of 1.8, and the ileum and jejunum are the most commonly involved sites. ${ }^{4}$ A case of SBM was reported in Korea; however, it was not primary SBM but secondary SBM. To the best of our knowledge, this is the first case of primary SBM in South Korea.

Generally, the small intestine and colon contain no melanocytes. There are some possible mechanisms to explain how malignant melanoma arises from the GI tract. Some experts believe that embryologically, melanocytes arise from melanoblastic neural crest cells that migrate via the omphalomesenteric canal to the distal ileum. ${ }^{10}$ Others suggest that these tumors originate from enteric neuroendocrine noncutaneous tissue in the form of amine precursor uptake decarboxylase (APUD) cells. ${ }^{11}$ Mishima explained that SBM might arise from neuroblastic Schwann cells associated with the autonomic innervation of the intestine. ${ }^{12}$ However, many experts believe that primary SBM does not exist, and secondary deposits from regressed or undiagnosed cutaneous melanoma are mistakenly considered primary SBM. ${ }^{4,8,13}$

Therefore, it is challenging to distinguish between primary and secondary SBM. Criteria for diagnosing primary SBM have been suggested, as follows, although they remain quite conjectural. The following should be present for a diagnosis of primary SBM: (1) a solitary lesion; (2) an intraluminal mass, with no other metastasis, excepting secondary regional mesenteric lymph nodes; (3) no evidence of disease in any other organs and no history of previous melanoma; and (4) a disease-free survival period of at least 12 months after surgical resection without any evidence of recurrence. ${ }^{6,14}$ The present case fulfilled these criteria, although potential regressed or unidentified ocular or extraintestinal tumor sites cannot be absolutely excluded. The clinical significance of this distinction lies within the differential prognosis. The prognosis is worse for primary SBM, which tends to grow faster and more aggressively than metastatic tumors, perhaps due to the rich lymphovascular supply available in the intestinal mucosa. ${ }^{15}$

Clinical diagnosis of SBM is difficult because its clinical symptoms and signs are nonspecific. The most common symptoms are abdominal pain, intestinal obstruction, occult or gross bleeding, fatigue, weight loss, and presence of a palpable abdominal mass. ${ }^{13}$ Rarely, intestinal melanoma presenting as single or multiple polypoid lesions can cause intestinal intussusception, as in the present case. ${ }^{16}$ This patient did not complain of any abdominal symptoms despite ileo-ileal intussusception.

Intestinal melanoma is typically diagnosed by radiologic examination, including abdominal CT, conventional barium contrast studies, or PET scan. However, the rate of clinical detection is low. ${ }^{13}$ Thus, a high index of suspicion is essential for diagnosis of the rare small bowel tumor. In the present case, abdominal CT had been performed two months prior to diagnosis, but no significant abnormalities had been detected on the CT scan. Retrospective review of the CT scan showed eccentric wall thickening in the distal ileum.

Conventional endoscopic examination of the small intestine is limited because the jejunum and ileum are not easily examined. In the majority of reported cases, diagnosis of small intestinal melanoma relied on radiologic examination and confirmation by exploratory laparotomy. ${ }^{3,1417-19}$ Recently, Hadjinicolaou et al. reported that they identified jejunal malignant melanoma by capsule endoscopy, and confirmative diagnosis was made after surgery. ${ }^{4}$ In the present case, we identified the location and shape of the ileal mass by single-balloon enteroscopy, and confirmed the diagnosis of melanoma preoperatively.

Single-balloon enteroscopy has several advantages compared with capsule endoscopy for the diagnosis of small bowel tumor, even though single-balloon enteroscopy is an invasive, technically difficult, and time-consuming procedure. First, single-balloon enteroscopy permits biopsy tissue to be obtained, permitting determination of a final diagnosis prior to surgery. Second, the procedure allows surgical site marking for better identification of the lesion during surgery. In addition, it can be used cautiously in patients with intestinal obstruction, as in the present case, whereas capsule endoscopy is contraindicated.

Regardless of the exact origin of the melanotic lesion of the small bowel, surgical resection with sufficient free margins is the treatment of choice. Surgery, if curative, has a low risk of surgical morbidity and mortality, and long-term disease-free survival can be expected. ${ }^{3}$ Chemotherapy, immunotherapy, and target therapy have a role in medical treatment of small intestinal melanoma but they are almost invariably used palliatively. No standard systemic therapies are available for patients with intestinal melanoma. ${ }^{4,6,13}$

In summary, we experienced a rare case of primary SBM, diagnosed by single-balloon enteroscopy. Primary SBM is a rare, solitary, intraluminal tumor without a history of melanoma in another organ. To confirm primary SBM, thorough 
investigation is required to identify a possible primary lesion.

\section{Conflicts of Interest}

The authors have no financial conflicts of interest.

\section{Acknowledgments}

This research was supported by grants from the Inha University Research Grant.

\section{REFERENCES}

1. Chang AE, Karnell LH, Menck HR. The national cancer data base report on cutaneous and noncutaneous melanoma: a summary of 84,836 cases from the past decade. The American College of Surgeons Commission on Cancer and the American Cancer Society. Cancer 1998;83:1664-1678.

2. Reintgen DS, Thompson W, Garbutt J, Seigler HF. Radiologic, endoscopic and surgical considerations of malignant melanoma metastatic to the small intestine. Curr Surg 1984;41:87-89.

3. Timmers TK, Schadd EM, Monkelbaan JF, Meij V. Survival after resection of a primary malignant melanoma of the small intestine in a young patient: report of a case. Case Rep Gastroenterol 2013;7:251-260.

4. Hadjinicolaou AV, Hadjittofi C, Athanasopoulos PG, Shah R, Ala AA. Primary small bowel melanomas: fact or myth? Ann Transl Med 2016;4:113.

5. Mihajlovic M, Vlajkovic S, Jovanovic P, Stefanovic V. Primary mucosal melanomas: a comprehensive review. Int J Clin Exp Pathol 2012;5:739753.

6. Blecker D, Abraham S, Furth EE, Kochman ML. Melanoma in the gastrointestinal tract. Am J Gastroenterol 1999;94:3427-3433.

7. Das Gupta TK, Brasfield RD. Metastatic melanoma of the gastrointestinal tract. Arch Surg 1964;88:969-973.
8. Schuchter LM, Green R, Fraker D. Primary and metastatic diseases in malignant melanoma of the gastrointestinal tract. Curr Opin Oncol 2000;12:181-185.

9. Kim W, Baek JM, Suh YJ, Jeon HM, Kim JA. Ileal malignant melanoma presenting as a mass with aneurysmal dilatation: a case report. J Korean Med Sci 2004;19:297-301.

10. Amar A, Jougon J, Edouard A, Laban P, Marry JP, Hillion G. [Primary malignant melanoma of the small intestine]. Gastroenterol Clin Biol 1992;16:365-367.

11. Krausz MM, Ariel I, Behar AJ. Primary malignant melanoma of the small intestine and the APUD cell concept. J Surg Oncol 1978;10:283288.

12. Mishima Y. Melanocytic and nevocytic malignant melanomas. Cellular and subcellular differentiation. Cancer 1967;20:632-649.

13. Lens M, Bataille V, Krivokapic Z. Melanoma of the small intestine. Lancet Oncol 2009;10:516-521.

14. Sachs DL, Lowe L, Chang AE, Carson E, Johnson TM. Do primary small intestinal melanomas exist? Report of a case. J Am Acad Dermatol 1999;41:1042-1044.

15. Liang KV, Sanderson SO, Nowakowski GS, Arora AS. Metastatic malignant melanoma of the gastrointestinal tract. Mayo Clin Proc 2006;81:511-516.

16. Kumari NS, Nandyala VNR, Devi KR, et al. Primary jejunal malignant melanoma presenting as intussusception: a rare case report. Int Surg J 2014;1:181-184.

17. Atmatzidis KS, Pavlidis TE, Papaziogas BT, Papaziogas TB. Primary malignant melanoma of the small intestine: report of a case. Surg Today 2002;32:831-833.

18. Butte JM, Meneses M, Waugh E, Parada H, De La Fuente H. Ileal intussusception secondary to small bowel metastases from melanoma. Am J Surg 2009;198:e1-e2.

19. Patti R, Cacciatori M, Guercio G, Territo V, Di Vita G. Intestinal melanoma: a broad spectrum of clinical presentation. Int J Surg Case Rep 2012;3:395-398. 